\title{
ON THE STRUCTURE OF SELF ADJOINT ALGEBRA OF FINITE STRICT MULTIPLICITY
}

\author{
S.C. ARORA and PAWAN BALA \\ Department of Mathematics \\ University of Delhi \\ Delhi-110 007, INDIA
}

(Received February 2, 1989, and in revised form April 30, 1989)

KEY WORDS AND PHRASES. Algebras of finite strict multiplicity, projections, self-adjoint, weakly closed algebra.

1980 AMS SUBJECT CLASSIFICATION CODES. Revision Primary 47C05, Secondary 47D25.

\section{INTRODUCTION.}

Throughout this paper $\mathrm{X}$ is a complex Hilbert space. For any subset $M$ of $X, B(M)$ denotes the algebra of all bounded linear operators on $M$. An algebra $\mathcal{A}$ means a strongly closed subalgebra of $B(X)$ containing the identity element $I . \mathcal{A}$ is said to be an algebra of finite strict multiplicity (a.f.s.m.), if there exists a finite subset $\Gamma=\left\{x_{1}, x_{2}, \ldots, x_{n}\right\}$ of $X$ such that $\mathcal{A}(\Gamma)=\left\{A_{1} x_{1}+A_{2} x_{2}+\ldots+A_{n} x_{n}, A_{i} \in \mathcal{A}\right\}=X$. In this case we denote the algebra by $\left(\mathcal{A},\left\{x_{i}\right\}_{i=1}^{n}\right)$. If $n=1$, i.e., if there exists a vector $x_{0}$ such that $\mathcal{A} x_{0}=\left\{A x_{0}: A \in \mathcal{A}\right\}=X$, then $\mathcal{A}$ is said to be a strictly cyclic algebra. In this case vector $x_{0}$ is called a strictly cyclic vector for $\ell$. Algebra $\mathcal{A}$ is said to be self-adjoint, if $A^{*} \in \mathcal{A}$, whenever $A$ is in $\mathcal{A}$. For any subset $\mathscr{B}$ of $B(X)$, the commutant of $\mathfrak{B}$, denoted by $\mathfrak{B}^{\prime}$, is the collection of all operators in $B(X)$ that commute with $\mathfrak{B}$.

A closed linear subspace $M$ of $X$ reduces the subset $\mathscr{B}$ of $B(X)$, if the projection of $X$ onto $M$ is in $\mathscr{B}^{\prime}$. A collection $\left\{M_{j}\right\}$ of closed linear subspaces of $X$ is said to be an orthogonal decomposition of $X$, if the $M j$ 's are pair-wise orthogonal and span $X$. Correspondingly, a collection $\left\{P_{j}\right\}$ of projections, is said to be a resolution of identity, if the collection $\left\{P_{j}(X)\right\}$ of ranges of $P_{i}$ forms an orthogonal decomposition of $X$.

Strictly cyclic operator algebras have been studied by Lambert [1], [2], M. Embry [3], [4], [5], Bolstein [6] and others. The study of strictly cyclic algebras was extended to that of algebras of finite strict multiplicity by Herrero in [7], [8]. This paper aims at studying the structure of the commutant of an a.f.s.m., particularly a self-adjoint a.f.s.m. in terms of its reducing subspaces. By [5], the commutant of a self-adjoint strictly cyclic algebra cannot have any infinite collection of pair-wise orthogonal projections. [9, Theorem 2] paves the way for the following:

2. MAIN RESULTS.

THEOREM 1. If $\left(\mathcal{A},\left\{x_{i}\right\}_{i=1}^{n}\right)$ is an a.f.s.m. on $X$, then each collection of mutually-orthogonal projections in $\mathcal{A}^{\prime}$ is finite. 
PROOF. Let $\left\{P_{j}\right\}$ be a collection of mutually-orthogonal projections in $\mathcal{A}^{\prime}$. We may assume $\left\{P_{j}\right\}$ to be countable. Let $Q_{n}=\sum_{j=1}^{n} P_{j}$ and $Q=\sum_{j=1}^{\infty} P_{j}$. $Q_{n}$ converges strongly to $Q$. By [9, Theorem 2], $Q_{n}$ converges uniformly to $Q$. As $Q-Q_{n}$ is a projection, its norm is zero or one. Since $\left\|Q_{n}-Q\right\|$ can be made arbitrarily small, there exists $m$ such that $\left\|Q_{n}-Q\right\|=0$ for all $n \geq m+1$. This implies that the collection $\left\{P_{j}\right\}=\left\{P_{j}\right\}_{j=1}^{m}$ is finite.

COROLLARY 2. Let $\left(\mathcal{A},\left\{x_{i}\right\}_{i=1}^{n}\right.$ be an a.f.s.m. on $X$. Any operator in $\mathcal{A}^{\prime}$ with residual spectrum empty is of finite spectrum.

PROOF. Let $E$ in $\mathcal{A}^{\prime}$ have residual spectrum empty. By [8], $E$ has no continuous spectrum. Therefore, spectrum of $E$ consists entirely of point spectrum. By Theorem $1, E$ has only finite number of distinct eigenspaces. So spectrum of $E$ is finite.

Our next theorem generalizes [5, Theorem 3] to a self-adjoint a.f.s.m.

THEOREM 3. Let $\mathcal{A}$ be a self-adjoint a.f.s.m. on $X$. Then there exists a finite orthogonal decomposition $\left\{M_{k}\right\}$ of $X$ such that each $M_{k}$ reduces $\mathcal{A}$, and $\left.\mathcal{A}\right|_{M_{k}}$ is strongly dense in $B\left(M_{k}\right)$.

PROOF. If $X$ and $\{0\}$ are the only reducing subspaces of $\mathcal{A}$, then by [8], $\mathcal{A}$ is strongly dense in $B(X)$. As such the trivial decomposition $\{X\}$ of $X$ satisfies the requirements of the theorem.

Let $\left\{M_{k}\right\}_{k=1}^{p}$ be a collection of mutually orthogonal subspaces of $X$ such that each $M_{k}$ reduces $\mathcal{A}$, and $\left.\mathcal{A}\right|_{M_{k}}$ is strongly dense in $B\left(M_{k}\right)$. If these $M_{k}$ 's span $X$, the theorem follows. Otherwise,

consider $A_{1}=\mathcal{A} \mid\left[\stackrel{P}{\stackrel{P}{V}} M_{i}\right]^{\perp}$. Let $P$ be orthogonal projection of $X$ onto $M=\left[\stackrel{P}{V} M_{i}\right]^{\perp}$. $P$ is in $\mathcal{A}^{\prime}$ and $\left(\mathcal{A},\left\{P x_{i}\right\}_{i=1}^{n}\right)$ is an a.f.s.m. on $M$. If $\mathcal{A}_{1}$ has only trivial reducing subspace, then again, by [8], $\mathcal{A}_{1}$ is strongly dense in $B(M)$ and the construction is complete. Otherwise, $\mathcal{A}_{1}$ has a non-trivial reducing subspace. This implies $\mathcal{A}_{1}$ has a minimal reducing subspace, say $M_{p+1}$. By [8], $\left.\mathcal{A}_{1}\right|_{M p+1}$ is strongly dense in $B\left(M_{p+1}\right)$. Thus, $M_{1}, M_{2}, \ldots, M_{p+1}$ are pair-wise orthogonal subspaces for $\mathcal{A}$, and $\left.\mathcal{A}\right|_{M_{k}}$ is strongly dense in $B\left(M_{k}\right)$ for $k=1,2, \ldots, p+1$. By Theorem 1, the collection terminates with a finite number of pair-wise orthogonal reducing subspaces.

Our next theorem depicts the structure of commutant of a self-adjoint a.f.s.m. The theorem and its consequences can be proved by following the technique used by Embry in [5]. So we omit the proofs.

THEOREM 4. Let $\left(\mathcal{A},\left\{x_{i}\right\}_{i=1}^{n}\right)$ be a self-adjoint a.f.s.m., $\left\{M_{k}\right\}$ a decomposition of $X$ as required in Theorem 3 and $P_{k}$ the orthogonal projection of $X$ onto $M_{k}$. Then $\mathcal{A}^{\prime}=\sum P_{j} \mathcal{A}^{\prime} P_{k}$ and $P_{j} \mathcal{A}^{\prime} P_{k}$ is of dimension one or zero for each value of $j$ and $k$. In particular, $\mathcal{A}^{\prime}$ is finitedimensional.

COROLLARY 5. If $\mathcal{A}$ is self-adjoint a.f.s.m. with an abelian commutant, then $\mathcal{A}^{\prime}=\left\{\sum_{j=1}^{n} \lambda_{j} P_{j}: \lambda_{j}\right.$ is complex $\}$, wherein $\left\{P_{j}\right\}$ are projections as required in Theorem 4. In particular, $\left\{P_{j}\right\}$ consists of normal operators with finite spectra.

COROLLARY 6. Let $N$ be a normal operator with $\{N\}^{\prime}$ as an a.f.s.m. Then there exist orthogonal projections $P_{1}, P_{2}, \ldots, P_{n}$ such that $\{N\}^{\prime \prime}=\left\{\sum_{=1}^{n} \lambda_{j} P_{j}, \lambda_{j}\right.$ complex $\}$.

COROLLARY 7. The decomposition $\left\{M_{k}\right\}$ in Theorem 4 is unique, if and only if, $\mathcal{A}^{\prime}$ is abelian.

If $\mathcal{A}$ is any a.f.s.m. on $X$, then $\mathcal{A}=\mathcal{A}_{1} \oplus \mathcal{A}_{2}$, where $\mathcal{A}_{1}$ is a self-adjoint a.f.s.m., and $\mathcal{A}_{2}$ is an a.f.s.m. having no reducing subspaces on which it is self-adjoint. 
An operator $T$ in $B(X)$ is said to be of finite strict multiplicity, if the weakly closed algebra $\mathcal{A}(T)$ generated by $T$ and $I$ is of finite strict multiplicity. Our next theorem extends [10, Theorem 6] proved by Barnes.

THEOREM 8. For an operator $T$ in $B(X)$, let $\left\{x_{1}, x_{2}, \ldots, x_{n}\right\}$ be a subset of $X$ such that $\left(\mathcal{A}(T),\left\{x_{i}\right\}_{i=1}^{n}\right)$ is an a.f.s.m. on $X$. Then there exists a finite mutually-orthogonal collection of subspaces $\left\{X_{1}, X_{2}, \ldots, X_{n}\right\}$ of $X$ satisfying the following

(i) Each $X_{i}$ reduces $T$

(ii) $X=X_{1} \oplus X_{2} \oplus \ldots \oplus X_{k}$ and thus $T=T_{1} \oplus T_{2} \ldots \oplus T_{k}$, where $T_{i}=\left.T\right|_{X_{i}}$.

(iii) Each $T_{j}$ is an irreducible operator of finite strict multiplicity on $X_{j}$.

PROOF. Let $\mathscr{B}$ be a closed subalgebra of $B(X)$ generated by $T, T^{*}$ and $I$. Define a positive functional $f$ on $\mathfrak{B}$ by $f: \mathfrak{B} \rightarrow C$ as $f(S)=\left(S x_{o}, x_{o}\right)$, where $x_{o}=x_{1}+x_{2}+\ldots+x_{n}, S$ in $\mathfrak{B}$. Let $K_{f}=\left\{S \in \mathfrak{B} ; f\left(S^{*} S\right)=0\right\}=\left\{S \in \mathfrak{B}: S x_{o}=0\right\}$. There are two norms on the quotient space $\mathfrak{B} / K_{f}$, viz.

(i ) $\left\|A+K_{f}\right\|_{f}=f\left(A^{*} A\right)^{1 / 2}=\left\|A x_{o}\right\|$

(ii) $\left\|A+K_{f}\right\|=\inf \left\{\|A-K\|: K \in K_{f}\right\}$.

These norms are related by $\left\|A+K_{f}\right\|_{f} \leq\left\|x_{o}\right\|\left\|A+K_{f}\right\|$ for all $A$ in $\mathscr{B}$. $\mathscr{B} / K_{f}$ is complete w.r.t. both these norms. By closed graph theorem, the norms are equivalent on $\mathbb{B} / K_{f}$. By Halpern [11], the commutant $\Im^{\prime}$ of $\Im_{B}$ in $B(X)$ has the following properties:

(i) If $F$ is a non-zero projection in $\mathscr{B}^{\prime}$, then $F$ majorizes a minimal projection $E$ in $\mathfrak{B}^{\prime}$;

(ii) A maximal set of mutually orthogonal projections in $\mathbb{B}$ must be finite.

By (i), we can choose a non-empty maximal set of mutually orthogonal projections in $\mathscr{B}^{\prime}$ and, by (ii), this set is finite. Let $\left\{E_{1}, E_{2}, \ldots, E_{k}\right\}$ be this set. Let $X_{j}=R\left(E_{j}\right), j=1,2, \ldots, k$. Then $X=X_{1} \oplus X_{2} \oplus \ldots \oplus X_{k}$. The collection $\left\{X_{1}, X_{2}, \ldots, X_{k}\right\}$ reduces $\mathfrak{B} ;$ and $\mathfrak{B}$ acts irreducibly on each $X_{j}, j=1,2, \ldots, k$. Now $x_{i} \in X$ implies $x_{i}=x_{i l} \oplus x_{i 2} \oplus \ldots \oplus x_{i k}, \iota=1,2, \ldots, n$ where $x_{i j} \in X_{j}$ for all $j=1,2, \ldots, k$. For $y \in X_{j} \subset X$, there exist operators $R_{1}, R_{2}, \ldots R_{n}$ in $A(T)$ such that

$$
\begin{aligned}
& y= R_{1} x_{1}+R_{2} x_{2}+\ldots+R_{n} x_{n} \\
&= R_{1}\left(x_{11} \oplus x_{12} \oplus \ldots \oplus x_{1 k}\right)+R_{2}\left(x_{21} \oplus \ldots \oplus x_{2 k}\right)+\ldots+R_{n}\left(x_{n 1} \oplus x_{n 2} \oplus \ldots \oplus x_{n k}\right) \\
&=\left(R_{1} x_{11}+R_{2} x_{21}+\ldots+R_{n} x_{n 1}\right) \oplus\left(R_{1} x_{12}+R_{2} x_{22}+\ldots+R_{n} x_{n 2}\right) \oplus \ldots \oplus \\
& \qquad\left(R_{1} x_{1 k}+R_{2} x_{2 k}+\ldots+R_{n} x_{n k}\right)
\end{aligned}
$$

As $T$ reduces $X_{j}, A(T)$ also reduces $X_{j}, j=1,2, \ldots, k$. This implies that

$$
y=R_{1} x_{1 j}+R_{2} x_{2 j}+\ldots+R_{n} x_{n} j=\left.R_{1}\right|_{X_{j}} x_{1 j}+\left.R_{2}\right|_{X_{j}} x_{2 j}+\ldots+\left.R_{n}\right|_{X_{j}} x_{n j},
$$

where $\left.R_{i}\right|_{X_{j}} \in A\left(T_{j}\right)$ for all $i=1,2, \ldots, n$. Thus $\left(A\left(T_{j}\right), x_{1 j}, x_{2 j}, \ldots, x_{n j}\right)$ is an a.f.s.m. on $X_{j}$.

This completes the proof of the theorem. 
ACKNOWLEDGMENT. The authors are thankful to Prof. B.S. Yadav for the inspiration received in carrying out this study.

\section{REFERENCES}

1. LAMBERT, A., Strictly Cyclic Operator Algebras, Pacific J. Math. 39 (1971) 717-726.

2. LAMBERT, A., Spectral Properties of Strictly Cyclic Operator Algebras, Indiana Univ. Math.

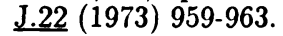

3. EMBRY, M.R., Strictly Cyclic Operator Algebras on Banach Space, Pacific J. Math 45 (1973) 445-452.

4. EMBRY, M.R., Maximal Invariant Subspaces of Strictly Cyclic Operator Algebras, Pacific. J. Math. $\underline{49}$ (1973) 45-50.

5. EMBRY, M.R., Self-adjoint Strictly Cyclic Operator Algebras, Pacific J. Math $\underline{52}$ (1974), 5357.

6. BOLSTEIN, R., Strictly Cyclic Operators, Duke Math J. 40 (1973) 683-688.

7. HERRERO, D.A., Algebras de Operadores Que contieven Una Subalgebra de Multiplicidad Estucta Finite, ㄹev. de la Union Mat. Argentina 26(1972), 77-83

8. HERRERO, D.A., Operator Algebras of Finite-Strict Multiplicity, Indrana Univ. Math. J.22 (1972) 13-24.

9. ARORA, S.C. and BALA, PAWAN, Invariant Subspaces of Algebras of Finite Strict Multiplicity. (Communicated).

10. BARNES, BRUCE, Operators With a Strictly Cyclic Vector, Proc. Amer. Math. Soc. 41 (1973), 480-486.

11. HALPERN, H., Finite Sums of Irreducible Functionals on C*-Algebras, Proc. Amer. Math. Soc. 18 (1967), 352-358. 


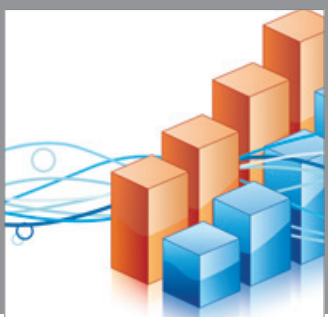

Advances in

Operations Research

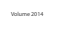

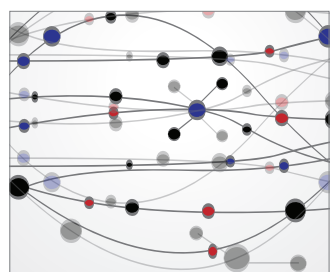

\section{The Scientific} World Journal
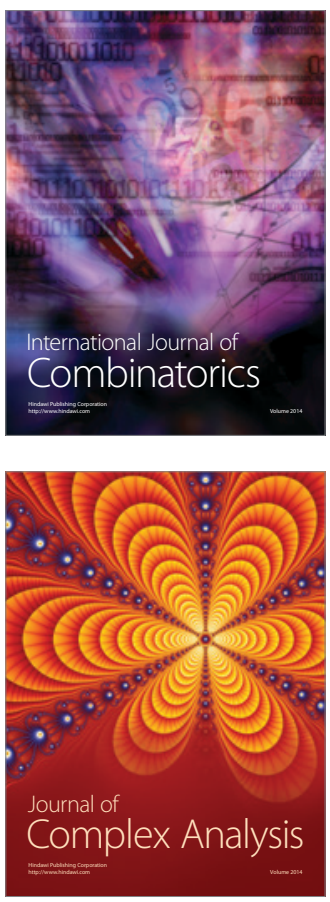

International Journal of

Mathematics and

Mathematical

Sciences
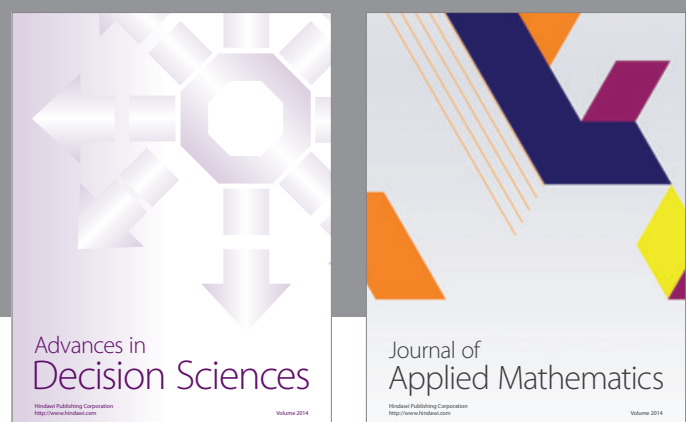

Journal of

Applied Mathematics
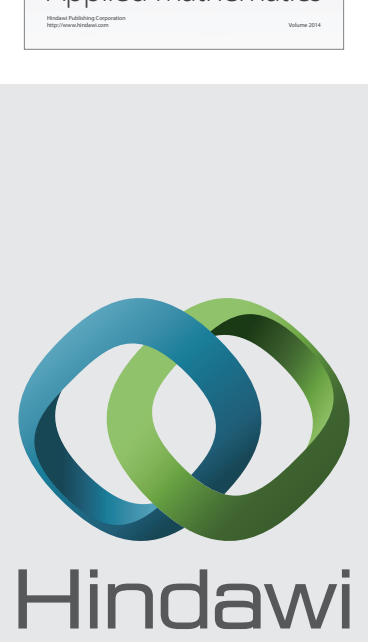

Submit your manuscripts at http://www.hindawi.com
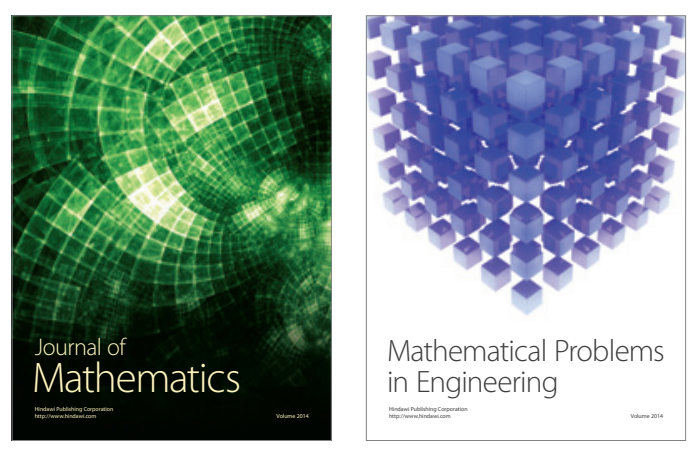

Mathematical Problems in Engineering
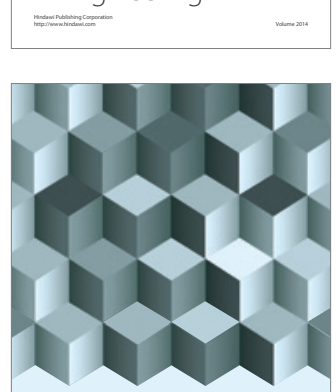

Journal of

Function Spaces
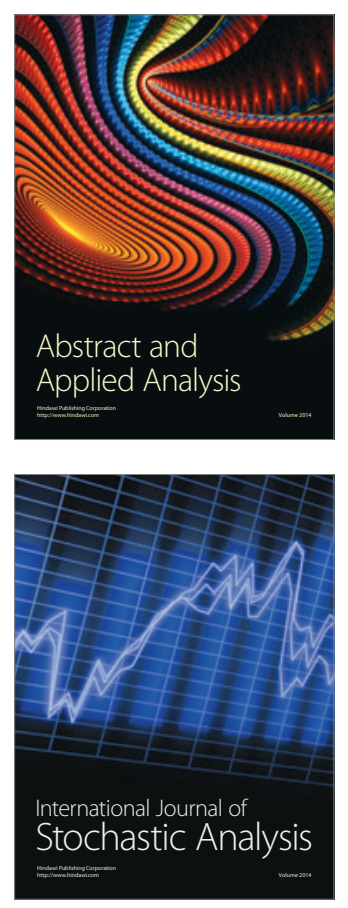

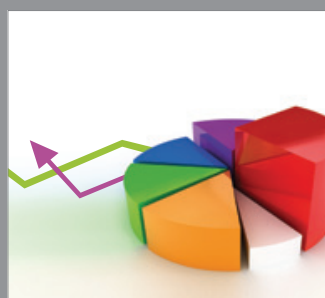

ournal of

Probability and Statistics

Promensencen
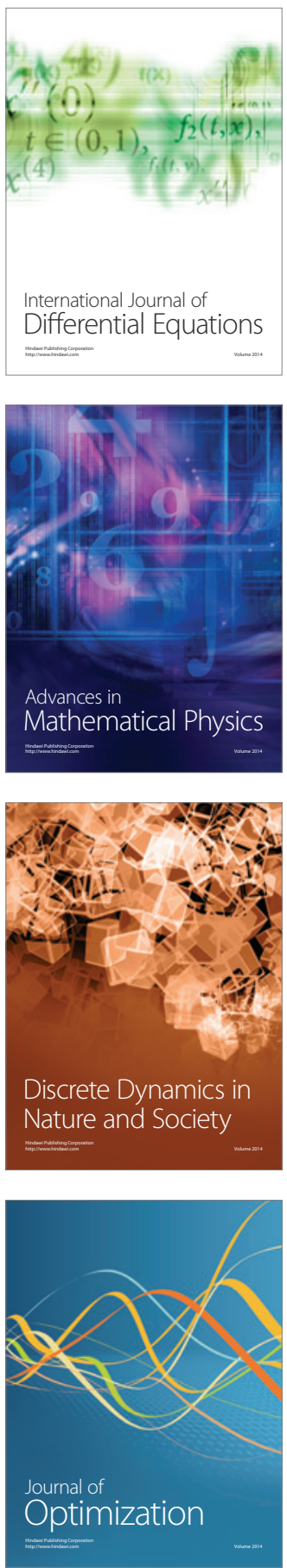